\title{
Microarray Analysis of Papillary Thyroid Cancers in Korean
}

\author{
Hyun Sook Kim', Do Hyung Kim², Ji Yeon Kim', Nam Ho Jeoung³, In Kyu Lee², Jin Gu Bong', and Eui Dal Jung'
}

\begin{abstract}
${ }^{1}$ Department of Internal Medicine, Catholic University of Daegu School of Medicine, Daegu; ${ }^{2}$ Department of Internal Medicine, Kyungpook National University School of Medicine, Daegu; ${ }^{3}$ Department of Fundamental Medical and Pharmaceutical Sciences, Catholic University of Daegu, Gyeongsan; ${ }^{4}$ Department of General Surgery, Catholic University of Daegu School of Medicine, Daegu, Korea
\end{abstract}

Background/Aims: Papillary thyroid cancer (PTC) is the most common malignancy of the thyroid gland. It involves several molecular mechanisms. The BRAF V600E mutation has been identified as the most common genetic abnormality in PTC. Moreover, it is known to be more prevalent in Korean PTC patients than in patients from other countries. We investigated distinct genetic profiles in Korean PTC through cDNA microarray analysis.

Methods: Transcriptional profiles of five PTC samples and five paired normal thyroid tissue samples were generated using cDNA microarrays. The tumors were genotyped for BRAF mutations. The results of the cDNA microarray gene expression analysis were confirmed by real-time PCR and immunohistochemistry analysis of 35 PTC patients.

Results: Four of the five patients whose PTC tissues were subjected to microarray analysis were found to carry the BRAF V600E mutation. Microarrays analysis of the five PTC tissue samples showed the expression of 96 genes to be increased and that of 16 genes decreased. Real-time reverse transcription-polymerase chain reaction (RT-PCR) confirmed increased expression of SLC34A2, TM7SF4, COMP, KLK7, and KCNJ2 and decreased expression of FOXA2, SLC4A4, LYVE-1, and TFCP2L1 in PTC compared with normal tissue. Of these genes, TFCP2L1, LYVE-1, and KLK7 were previously unidentified in PTC microarray analysis. Notably, Foxa2 activity in PTC was reduced, as shown by its cytoplasmic localization, in immunohistochemical analyses.

Conclusions: These findings demonstrate both similarities and differences between our results and previous reports. In Korean cases of PTC, Foxa2 activity was reduced with its cytoplasmic accumulation. Further studies are needed to confirm the relationship between FOXA2 and BRAF mutations in Korean cases of PTC. (Korean $\mathbf{J}$ Intern Med 2010;25:399-407)

Keywords: BRAF mutation; Oligonucleotide array sequence analysis; FOXA2 protein, human; Thyroid cancer

\section{INTRODUCTION}

Papillary thyroid cancer (PTC) is the most common thyroid cancer in iodine-sufficient regions [1]. It is believed to account for more than $80 \%$ of all thyroid cancers $[2,3]$. The clinical characteristics of PTC are diverse, ranging from slow, progressive micro-PTC to anaplastic cancers [4,5].

Although the pathogenetic mechanisms behind PTC remain unknown, multiple genes and environmental factors have been implicated in its development $[3,6]$. $R E T / P T C$ rearrangements, $R A S$ mutations, and $B R A F$ mutations are known genetic abnormalities in PTC. The incidences of $R E T / P T C$ rearrangements, $R A S$ mutations, and $B R A F$ mutations in PTC are $13-43 \%, 0-21 \%$, and 29$69 \%$, respectively [7]. In Korean PTC patients, $R A S$ mutations are rare, and $B R A F$ mutation more common [8].

The gold standard method for diagnosing PTC is fineneedle aspiration biopsy (FNAB) [9,10]. Nevertheless, 10-

Received: May 18, 2010

Revised : August 12, 2010

Accepted: September 15, 2010 
$15 \%$ of $\mathrm{FNAB}$ results are inadequate, and $10-20 \%$ indeterminate, making accurate diagnosis of the thyroid nodules difficult [11].

To manage the limitations of FNAB in PTC, many researchers have attempted to identify useful genetic tools. One such tool, DNA microarray analysis, is expected to provide information about the pathogenetic mechanism and genetics of PTC. According to a metaanalysis of 21 published thyroid cancer gene expression profiling studies, in addition to genes such as $M E T, T F F 3$, SERPINA1, TIMP1, FN1, and TPO that have previously been linked to PTC, other genes such as TGFA, OPCT, CRABP1, FCGBP, EPS8, and PROS1 showed different expression between PTC samples and normal tissues [12]. In a separate microarray analysis, PTC cases with $R E T / P T C$ rearrangements, $R A S$ mutations, and $B R A F$ mutations displayed distinct gene expression profiles [13].

The present study was undertaken in Korean PTC patients, who showed a high prevalence of BRAF mutations. Our objective was to identify, in microarray analyses, genes whose expression was altered in Korean PTC as candidate diagnostic markers in PTC.

\section{METHODS}

\section{Subjects}

PTC tissue samples were obtained intra-operatively from 35 patients during thyroidectomy procedures performed between January 2008 and December 2008. Normal tissue samples were collected from the same patients for pair-wise analysis. The study protocol was approved by the Institutional Review Board, and all patients signed informed consent.

\section{Isolation of RNA and DNA microarray analysis}

Tissue samples obtained during thyroidectomy were immediately frozen in liquid nitrogen and stored. Total RNA was extracted using TRIzol (Invitrogen, Camarillo, CA, USA) and purified using an RNeasy Mini Kit (Qiagen, Hilden, Germany). Sample purity was confirmed by measuring $\mathrm{A}_{260} / \mathrm{A}_{280}$ ratios, and quality by $1 \%$ agarose gel electrophoresis. RNA samples from five PTC tissue/normal tissue pairs were chosen for microarray studies. Biotinylated cRNA was prepared from $0.55 \mu \mathrm{g}$ of total RNA using the Illumina TotalPrep RNA Amplification Kit (Ambion, Austin, TX, USA). Following fragmentation, $0.75 \mu \mathrm{g}$ of cRNA was hybridized to
Illumina HumanHT-12 v3 Expression BeadChips (Illumina Inc., San Diego, CA, USA) according to protocols provided by the manufacturer.

\section{Real-time RT-PCR analysis}

The expression of genes identified in microarray analyses as being up-regulated ( $\mathrm{TM}_{7} \mathrm{SF}_{4}, \mathrm{SLC}_{34} \mathrm{~A}_{2}$, $K_{C N J} 2, C O M P$, and $\left.K L K_{7}\right)$ and down-regulated (TFCP2L1, LYVE-1, FOXA2, and SL4A4) in PTC was examined by real-time reverse transcription-polymerase chain reaction (RT-PCR). A RevertAid ${ }^{\mathrm{TM}}$ First Strand cDNA Synthesis Kit (Fermentas, Hanover, MD, USA) was used to synthesize cDNA. PCR reactions (20 mL) contained total RNA ( $2 \mathrm{mg}$ ), oligo (dT) 18 primer ( $1 \mathrm{~mL}$ ), 5 $\times$ reaction buffer $(4 \mathrm{~mL})$, RiboLock ${ }^{\mathrm{TM}}$ Ribonuclease Inhibitor (20 u/mL) (1 mL), $10 \mathrm{mM} \mathrm{dNTP} \mathrm{mix} \mathrm{(2} \mathrm{mL),}$ and RevertAid ${ }^{\mathrm{TM}}$ M-MuLV Reverse Transcriptase (200 $\mathrm{u} / \mathrm{mL}$ ) (1 mL). An ABI PRISM 7500 Sequence Detection System (Applied Biosystems, Foster City, CA, USA) and TaqMan probe PCR master mix (Applied Biosystems) were used in real-time PCR reaction. The PCR amplification procedure was carried out in 96-well plates in 20-mL reactions containing cDNA $(1 \mu \mathrm{L})$, probe $(1 \mu \mathrm{L})$, Master Mix $(10 \mu \mathrm{L})$ and QW $(8 \mu \mathrm{L})$. The following thermal conditions were applied: $50^{\circ} \mathrm{C}$ for 2 minutes; $95^{\circ} \mathrm{C}$ for 10 minutes; and 40 cycles of $95^{\circ} \mathrm{C}$ for 15 seconds and $60^{\circ} \mathrm{C}$ for 1 minute.

\section{BRAF mutation analysis}

$B R A F$ mutation analysis was performed using the five papillary thyroid cancer tissue samples subjected to microarray analysis. Genomic DNA was extracted using G-DEX (iNtRON Biotechnology Co. Ltd., Seoul, Korea). Exon 15 of $B R A F$ was PCR-amplified using the following PCR primers: $B R A F 15,5^{\prime}$ - ATGTTGCTCTGATAGGAAA $-3^{\prime}$ (sense), 5' - GATTTTTGTGAATACTGGGAA -3' (antisense).

\section{Statistical analysis}

Significant differences in gene expression between cancerous and normal tissues in the microarray analysis were identified using paired Student's $t$ test, fold-change, and hierarchical clustering, performed using SPSS version 14.0 (SPSS Inc., Chicago, IL, USA). Differences were considered statistically significant when the falsediscovery rate (FDR) was $10 \%$ of the control ( $p$ values were subjected to Benjamini-Hochberg FDR correction) and the fold-change was $\geq 2$. 


\section{RESULTS}

\section{Clinical characteristics of the study patients}

The mean patient age was $50.2 \pm 13.2$ years. The patient group comprised 3 men and 32 women. The classic PTC subtype was found in 34 patients, and the follicular variant in one patient. The mean tumor size was $1.3 \pm 0.6$ $\mathrm{cm}$. Eighteen cases (50\%) showed extrathyroidal invasion, and 22 cases (66\%) showed lymph node metastasis.

\section{BRAF mutations and gene expression in DNA microarray analysis}

Four of the five PTC samples subjected to microarray analysis were positive for the T1799A BRAF mutation. DNA microarray analysis of 5 PTC samples identified 112 genes whose expression showed a more than 2-fold change and where FDR was 10\% of the control in PTC tissue compared with paired normal tissue. Of these, 96 genes, including $\mathrm{TM}_{7} \mathrm{SF}_{4}$, SLC34A2, KCNJ2, C7orf24, PROS1, SERPINA1, TUSC3, COMP, kallikrein-related peptidase $7(K L K 7)$, and matrix metallopeptidase 7 , showed more than 2-fold higher expression in PTC tissues compared with paired normal tissues (Table 1). Expression of the remaining 16 genes, which included metallothionein 1F (MT1F), HBA2, ACACB, RYR2, OTOS, SLC4A4, TFCP2L1, LYVE-1, and FOXA2, was decreased more than 2-fold in PTC tissues compared with paired normal tissue (Table 1).

Of the genes previously linked to $B R A F$ mutationassociated PTC, TM7SF4, SLC34A2, PDE5A, TPD52L1, $F N 1, P L X N C 1, T M P R S S 6$, and ERBB3 displayed more than 2-fold increased expression in PTC tissues, whereas the expression of $A R N T L, P E R P, G A T A 3, I R S 2$, CLECSF2, and STAT1 was increased less than 2-fold in PTC tissues compared with paired normal tissues (Table 2). The expression of $T F F 3, A R G B P 2, H G D$, FHL1, IRS1,

Table 1. Up-regulated or down-regulated genes in microarray analysis of papillary thyroid cancer

\begin{tabular}{lc}
\hline Gene & Fold change \\
\hline Transmembrane 7 superfamily member 4 (TM7SF4) & 20.22 \\
Solute carrier family 34 (sodium phosphate), member 2 (SLC34A2) & 14.56 \\
Serpin peptidase inhibitor, clade A (alpha-1 antiproteinase, antitrypsin), member 1 (SERPINA1) & 13.25 \\
Cartilage oligomeric matrix protein (COMP) & 11.08 \\
Matrix metallopeptidase 7 & 8.67 \\
Kallikrein-related peptidase 7 (KLK7) & 7.01 \\
Protein S (alpha) (PROS1) & 5.73 \\
Potassium inwardly-rectifying channel, subfamily J, member 2 (KCNJ2) & 5.69 \\
Chromosome 7 open reading frame 24 (C7orf24) & 4.27 \\
Tumor suppressor candidate 3 (TUSC3) & 4.21 \\
Metallothionein 1F & -4.32 \\
Otospiralin (OTOS) & -4.11 \\
Forkhead box A2 (FOXA2) & -4.04 \\
Lymphatic vessel endothelial hyaluronan receptor 1 (LYVE-1) & -3.82 \\
Tanscription factor CP2-like 1 (TFCP2L1) & -3.76 \\
Solute carrier family 4, sodium bicarbonate cotransporter, member 4 (SLC4A4) & -3.13 \\
Ryanodine receptor 2 (cardiac) (RYR2) & -2.79 \\
Hemoglobin, alpha 2 (HBA2) & -2.51 \\
Acetyl-Coenzyme A carboxylase beta (ACACB) & -2.04 \\
\hline & \\
\hline
\end{tabular}


Table 2. Genes showing different expressions in this study among known distinct genes for BRAF mutant papillary thyroid cancer

\begin{tabular}{|c|c|}
\hline Gene & Fold change \\
\hline Transmembrane 7 superfamily member 4 (TM7SF4) & 20.22 \\
\hline Solute carrier family 34 (sodium phosphate), member 2 (SLC34A2) & 14.56 \\
\hline Transmembrane protease, serine 6 (TMPRSS6) & 7.65 \\
\hline Fibronectin 1 (FN1) & 5.99 \\
\hline V-erb-b2 erythroblastic leukemia viral oncogene homolog 3 (avian) (ERBB3) & 4.41 \\
\hline Aryl hydrocarbon receptor nuclear translocator-like (ARNTL) & 2.84 \\
\hline Tumor protein D52-like 1 (TPD52L1) & 2.64 \\
\hline Phosphodiesterase 5A (PDE5A) & 2.53 \\
\hline Plexin C1 (PLXNC1) & 2.26 \\
\hline TP53 apoptosis effector (PERP) & 1.86 \\
\hline Insulin receptor substrate 2 (IRS2) & 1.60 \\
\hline Signal transducer and activator of transcription $1,91 \mathrm{kDa}$ (STAT1) & 1.43 \\
\hline GATA binding protein 3 (GATA3) & 1.08 \\
\hline C-type lectin domain family 2, member B (CLECSF2) & 1.07 \\
\hline Trefoil factor 3 (intestinal) (TFF3) & -8.50 \\
\hline Sorbin and $\mathrm{SH} 3$ domain containing 2 (SORBS2: ARGBP2) & -3.67 \\
\hline Homogentisate oxidase $(H G D)$ & -3.25 \\
\hline Four and a half LIM domains 1 (FHL1) & -2.76 \\
\hline Insulin receptor substrate 1 (IRS1) & -2.50 \\
\hline Hepatic leukemia factor $(H L F)$ & -2.01 \\
\hline Matrix metallopeptidase 15 (MMP15) & -1.48 \\
\hline Slit homolog 3 (SLIT3: ARHN) & -1.47 \\
\hline Collagen, type IV, alpha 5 (COL4A5) & -1.16 \\
\hline Vav 3 guanine nucleotide exchange factor (VAV3) & -1.15 \\
\hline Phospholipase A2, group IVB (PLA2G4B) & -1.15 \\
\hline Hairy and enhancer of split 1, (Drosophila) (HES1) & -1.04 \\
\hline Dual oxidase $1(D \cup O X 1)$ & -1.04 \\
\hline UDP-N-acetyl-alpha-D-galactosamine:polypeptide N-acetylgalactosaminyltransferase 10 (GALNT10) & -1.03 \\
\hline
\end{tabular}

and $H L F$ was decreased more than 2-fold in PTC tissues compared with paired normal tissues, and that of GALNT1O, HES1, VAV3, MMP 15, PLA2G4B, COL4A5, $A R H N$, and DUOX1 less than 2-fold (Table 2).

\section{Functional classification of differently expressed genes}

We summarized the functional classifications of genes that were differently expressed in PTC tissues and paired 
Table 3. Functional classification of genes showing altered expression in papillary thyroid cancer

\begin{tabular}{|c|c|}
\hline Function & Genes \\
\hline Cell cycle & $\begin{array}{l}\text { Up-regulated: } K L K 7, T G F A, \text { Cyclin } D 1 \\
\text { Down-regulated: FOXA2 }\end{array}$ \\
\hline Cell adhesion molecule & Up-regulated: COL8A1, cadherin $3, N R C A M$, periostin \\
\hline Ion channel & $\begin{array}{l}\text { Up-regulated: KCNJ2, SCN-1B, SLC34A2, claudin } 16 \\
\text { Down-regulated: Ryanodine receptor-2 (RYR2) }\end{array}$ \\
\hline Nuclear acid binding & $\begin{array}{l}\text { Up-regulated: } R X R G, B H L H B 3, I R X 2, Z M A T 3 \\
\text { Down-regulated: forkhead box A2 (FOXA2) }\end{array}$ \\
\hline Receptor & $\begin{array}{l}\text { Up-regulated: } L P A R 5, A D O R A 1, X P R 1, R X R G \\
\text { Down-regulated: RYR2 }\end{array}$ \\
\hline Signaling molecule & $\begin{array}{l}\text { Up-regulated: } M D K, T G F A, C O M P, C B L N 1 \\
\text { Down-regulated: SEMA6A }\end{array}$ \\
\hline Transcription factor & $\begin{array}{l}\text { Up-regulated: IRX2, RXRG, RUNX1, ETV5, TSC22D1 } \\
\text { Down-regulated: TFCP2L1 }\end{array}$ \\
\hline Transporter & Up-regulated: SLC34A2 \\
\hline
\end{tabular}

normal tissues (Table 3). Of the genes involved in cellcycle control, the expression of $K L K 7, T G F A$, and cyclin D1 was increased, whereas that of FOXA2 decreased. Meanwhile, of the ion-channel genes, the expression of $S L C 34 A 2, K C N J 2$, and claudin 16 was increased, and that of $R Y R 2$ decreased. Expression of the adhesion molecules cadherin 3, NRCAM, POSTN, and ITGA2 was increased.

\section{Real-time RT-PCR analysis of gene expression}

From the genes displaying large differences in expression between PTC and normal tissues in the microarray analysis, we selected 9 for which information was available in PubMed. Microarray analysis showed the expression of $\mathrm{TM}_{7} \mathrm{SF}_{4}, \mathrm{SLC}_{34 \mathrm{~A} 2}, \mathrm{KCNJ} 2, \mathrm{COMP}$, and $K L K 7$ to be increased, and that of TFCP2L1, LYVE-1, FOXA2, and $\mathrm{SLC}_{4} 44$ to be decreased. Real-time RT-PCR analysis confirmed all these findings (Figs. 1 and 2).

\section{Immunohistochemical analysis of FOXA2, COMP, and LYVE-1}

We studied the products of the FOXA2, COMP, and $L Y V E-1$ genes immunohistochemically using commercially available antibodies. LYVE1 and COMP were found to be only weakly expressed and could not be fully analyzed. Foxa2 exhibited cytoplasmic expression in PTC tissues and nuclear expression in paired normal tissues (Fig. 3).

\section{DISCUSSION}

PTCs exhibit different patterns of gene expression according to the presence of $R E T / P T C$ rearrangements, $R A S$ mutations, and BRAF mutations. In the present study, we used DNA microarrays to analyze gene expression in Korean PTC patients, in whom the prevalence of $B R A F$ mutations is approximately $80 \%$ [8]. The results showed that the expression of 96 genes, including $\mathrm{TM}_{7} \mathrm{SF}_{4}, \mathrm{SLC}_{34} \mathrm{~A} 2, \mathrm{KCNJ} 2$, and $K L K_{7}$, was increased more than 2-fold in PTC tissues compared with paired normal tissues, and that of 16 genes, including MT1F, FOXA2, TFCP2L1, and LYVE-1, decreased more than 2-fold. Eszlinger et al. [14] previously identified shared and distinct gene expression in PTC subtypes characterized by $R E T / P T C, B R A F$, and $R A S$ mutations. They identified 20 up- and down-regulated genes in $B R A F$ 


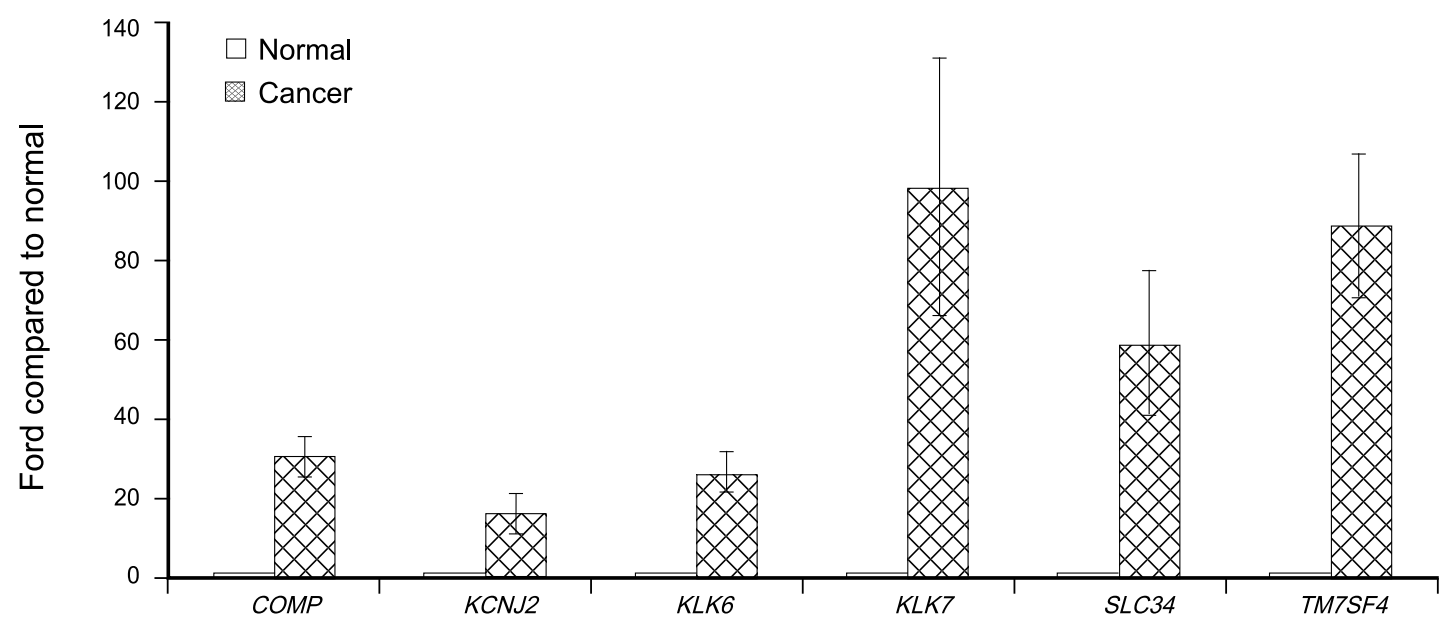

Figure 1. Real-time reverse transcriptase-polymerase chain reaction (RT-PCR) of genes that were up-regulated in papillary thyroid cancer tissues compared with paired normal tissues. COMP, cartilage oligomatrix protein; KCNJ2, potassium inwardly-rectifying channel, subfamily J, member 2; KLK-6, kallikrein-related peptidase 6; KLK-7, kallikrein-related peptidase 7; SLC 34, solute carrier family 34 (sodium phosphate); $T M 7 S F 4$, transmembrane 7 superfamily member 4.

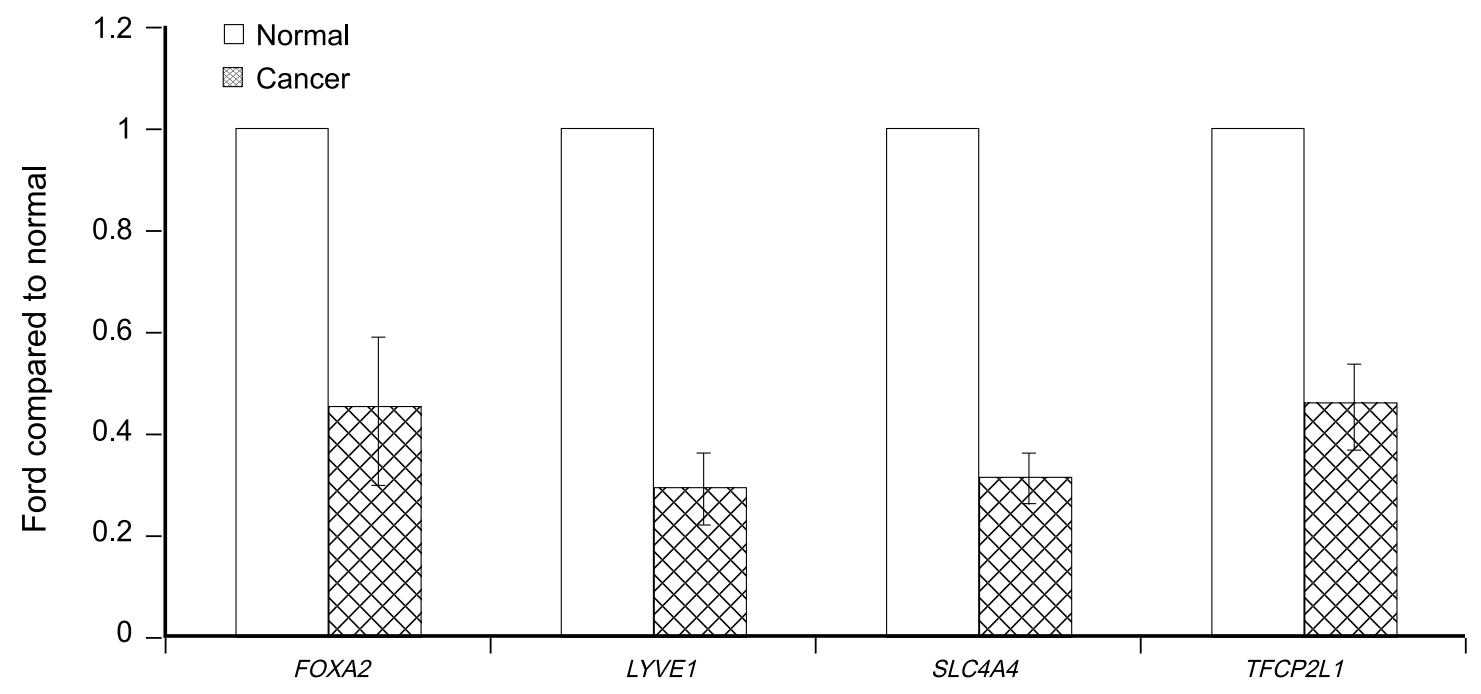

Figure 2. Real-time reverse transcriptase-polymerase chain reaction (RT-PCR) of genes that were down-regulated in papillary thyroid cancer tissues compared with paired normal tissues. FOXA2, forkhead box A2; LYVE-1, lymphatic vessel endothelial hyaluronan receptor 1; SLC4A4, solute carrier family 4, sodium bicarbonate cotransporter, member 4; TFCP2L1, transcription factor CP2-like 1.

mutation-associated PTC. Of these genes, we confirmed the expression of TM7SF4, SLC34A2, PDE5A, TPD52L1, FN1, PLXNC1, TMPRSS6, and ERBB3 to be increased more than 2-fold in PTC tissues compared with paired normal tissues, but found the expression of ARNTL,
PERP, GATA3, IRS2, CLECSF2, and STAT1 to be increased less than 2-fold in PTC tissues (Table 2). We also found similar $>$ 2-fold down-regulation of $T_{F F}$, $A R G B P 2, H G D, F H L 1, I R S 1$, and HLF in PTC tissues compared with paired normal tissues, but found the 

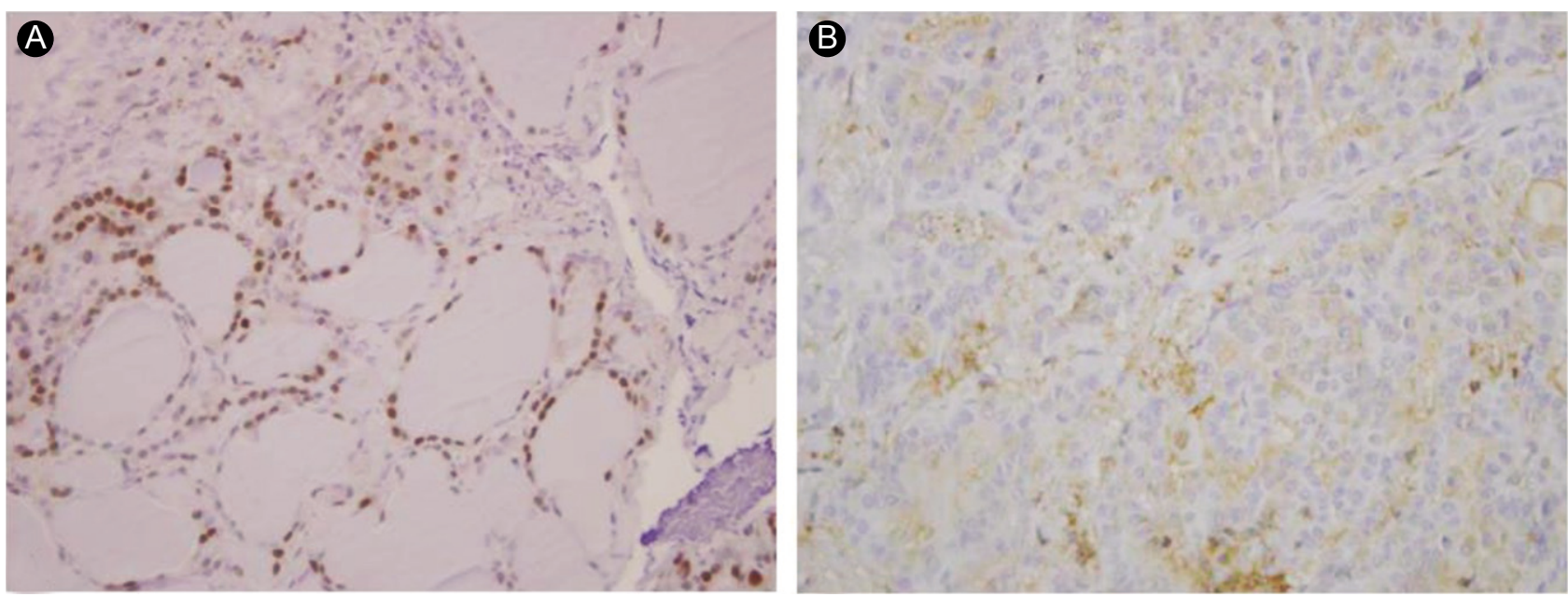

Figure 3. Cytoplasmatic accumulation of forkhead box A2 (FOXA2) in papillary thyroid cancer (PTC). (B) PTC tissues show especially cytoplasmatic accumulation, but (A) paired normal tissues show nuclear accumulation in Foxa2 antibody staining $(\times 100)$.

expression of GALNT1o, HES1, VAV3, MMP 15, $P L A 2 G 4 B, C O L 4 A 5, A R H N$, and DUOX1 to be decreased less than 2-fold in PTC tissues compared with paired normal tissues (Table 2). Moreover, although we found that the LYVE1, MT1F, OTOS, and FOXA2 expression was significantly decreased in PTC tissues compared with normal tissues, these genes were not among those showing different expression in the study of $B R A F$ mutation-associated PTC by Eszlinger et al. [14].

Our study has certain limitations. We did not specifically measure gene expression in $B R A F$ mutationpositive PTC. However, four of the five PTC samples that we subjected to microarray analysis were positive for $B R A F$ mutations. We therefore consider that our microarray results show characteristic gene expression profiles for Korean BRAF mutant PTC.

To confirm our initial findings, we analyzed a subset of genes showing altered expression in the microarray analysis by real-time RT-PCR. The results of this real-time RT-PCR confirmed the findings of the microarray analysis, showing the expression of $T_{M 7 S F}, K C N J 2$, $S L C 34 A 2, K L K 7$, and COMP to be increased in PTC tissues compared with normal tissues and that of FOXA2, $L Y V E-1, T F C P 2 L 1$, and SLC4A4 to be decreased.

First identified in dendritic cells [15], TM7SF4 (DC$S T A M P$ ) is also expressed in osteoclasts and is required for osteoclast cell fusion and monocyte-derived multinucleated giant cell formation [16]. A previous study employing the GeneFishing method reported that expression of the TM7SF4 and COL1A1 genes was significantly different in PTC tissues compared with normal tissues [17]. In addition, Galeza-Kulik et al. [18] analyzed tissue from 38 PTC patients by quantitative realtime PCR and identified several genes involved in the transport of ions whose expression was altered in PTC. They reported increased $S L C 34 A 2$ and $K C N J 2$ expression and reduced $S L C 4 A 4$ expression in PTC tissues. The results of our microarray and real-time RT-PCR analyses confirmed the expression of $S L C 34 A 2$ and $K C N J 2$ to be increased and that of SLC4A4 decreased in PTC tissues. The kallikrein (KLK) family comprises 15 structurally homogeneous, trypsin-like serine protease genes [19]. KLK4 and KLK11 are known to be overexpressed in ovarian cancer and prostate cancer, and KLK6 to be down-regulated in breast cancer and prostate cancer [20]. Talieri et al. [21] found that $K L K 7$ was more highly expressed in colon cancer than in normal tissues, and they proposed that it may be a prognostic factor in colon cancer patients. In the present study, microarray analysis showed $K L K 7$ expression to be 7 -fold higher in PTC tissues than in normal tissues, a result that was confirmed by real-time RT-PCR. Differences in KLK family gene expression in PTC have not previously been studied and warrant further research.

Members of the forkhead box-O (FOXO) family of proteins are known to regulate important cellular events such as differentiation, DNA repair, cell cycle arrest, and apoptosis [22,23]. Karger et al. [24] reported the cytoplasmic accumulation of Foxo3a in differentiated thyroid cancer, in contrast to its nuclear accumulation in normal thyroid tissue and follicular adenoma. Cytoplasmic accumulation of Foxo3a results in increased phospho-activation of Akt, decreased transcription of the Foxo3a target genes p27kip and Bim, and increased 
expression of Gadd45a mRNA [24]. The authors concluded that the inactivation of Foxo3a may represent a pathogenetic mechanism for the avoidance of cancer cell apoptosis in thyroid follicular carcinoma. Separately, Akagi et al. [25] reported decreased expression of FOXA2 in PTC cell lines as a result of methylation of CpG islands in the FOXA2 promoter. They also reported that forced expression of FOXA2 inhibited cancer cell growth in PTC. Similarly, in the present study, we showed through microarray analysis and real-time RT-PCR that FOXA2 expression was decreased in PTC tissues compared with normal tissues. In immunohistochemical analyses, we showed that Foxa2 accumulated in the cytoplasm of PTC tissues and in the nucleus in normal tissues. This suggests that Foxa2 is transported from the nucleus to the cytoplasm in PTC. Further study is required to determine whether the cause of decreased FOXA2 expression is marked cytoplasmic accumulation of FOXA2 or the methylation of $\mathrm{CpG}$ islands in the promoter region of FOXA2.

This study has certain limitations. First, the number of subjects was small. Second, the microarray analysis may not have been optimal. It is still not clear which preprocessing algorithm is the most appropriate, and some researchers argue that the statistical tools used to analyze microarray analysis results remain insufficient. Third, we did not test whether gene expression changes translated into changes in protein expression. Thus, additional studies that include a larger number of subjects and that analyze expression at the protein level are necessary.

In conclusion, our study confirmed some results of previous studies results and yielded others that were different. Our results show that FOXA2 is down-regulated in Korean cases of PTC as a result of the transport of Foxa2 from the nucleus to the cytoplasm. These findings provide insights into the molecular pathways involved in PTC in the Korean population.

\section{Conflict of interest}

No potential conflict of interest relevant to this article was reported.

\section{Acknowledgements}

This work was supported by National Research
Foundation of Korea Grant funded by the Korean Government (KRF-2008-331-Eoo125).

\section{REFERENCES}

1. Lind P, Langsteger W, Molnar M, Gallowitsch HJ, Mikosch P, Gomez I. Epidemiology of thyroid diseases in iodine sufficiency. Thyroid 1998;8:1179-1183.

2. Hundahl SA, Fleming ID, Fremgen AM, Menck HR. A National Cancer Data Base report on 53,856 cases of thyroid carcinoma treated in the U.S., 1985-1995. Cancer 1998;83:2638-2648.

3. Gimm O. Thyroid cancer. Cancer Lett 2001;163:143-156.

4. Neff RL, Farrar WB, Kloos RT, Burman KD. Anaplastic thyroid cancer. Endocrinol Metab Clin North Am 2008;37:525-538, xi.

5. McIver B, Hay ID, Giuffrida DF, et al. Anaplastic thyroid carcinoma: a 50-year experience at a single institution. Surgery 2001;130:1028-1034.

6. Kinder BK. Well differentiated thyroid cancer. Curr Opin Oncol 2003;15:71-77.

7. Kondo T, Ezzat S, Asa SL. Pathogenetic mechanisms in thyroid follicular-cell neoplasia. Nat Rev Cancer 2006;6:292-306.

8. Kim KH, Kang DW, Kim SH, Seong IO, Kang DY. Mutations of the BRAF gene in papillary thyroid carcinoma in a Korean population. Yonsei Med J 2004;45:818-821.

9. Slough CM, Randolph GW. Workup of well-differentiated thyroid carcinoma. Cancer Control 2006;13:99-105.

10. Amrikachi M, Ramzy I, Rubenfeld S, Wheeler TM. Accuracy of fine-needle aspiration of thyroid. Arch Pathol Lab Med 2001;125:484-488.

11. Goellner JR, Gharib H, Grant CS, Johnson DA. Fine needle aspiration cytology of the thyroid, 1980 to 1986 . Acta Cytol 1987;31:587-590.

12. Griffith OL, Melck A, Jones SJ, Wiseman SM. Meta-analysis and meta-review of thyroid cancer gene expression profiling studies identifies important diagnostic biomarkers. J Clin Oncol 2006;24:5043-5051.

13. Giordano TJ, Kuick R, Thomas DG, et al. Molecular classification of papillary thyroid carcinoma: distinct BRAF, RAS, and RET/PTC mutation-specific gene expression profiles discovered by DNA microarray analysis. Oncogene 2005;24:6646-6656.

14. Eszlinger M, Krohn K, Kukulska A, Jarzab B, Paschke R. Perspectives and limitations of microarray-based gene expression profiling of thyroid tumors. Endocr Rev 2007;28:322-338.

15. Hartgers FC, Vissers JL, Looman MW, et al. DC-STAMP, a novel multimembrane-spanning molecule preferentially expressed by dendritic cells. Eur J Immunol 2000;30:3585-3590.

16. Yagi M, Miyamoto T, Sawatani Y, et al. DC-STAMP is essential for cell-cell fusion in osteoclasts and foreign body giant cells. J Exp 
Med 2005;202:345-351.

17. Lee KY, Huang SM, Li S, Kim JM. Identification of differentially expressed genes in papillary thyroid cancers. Yonsei Med J 2009;50:60-67.

18. Galeza-Kulik M, Zebracka J, Szpak-Ulczok S, et al. Expression of selected genes involved in transport of ions in papillary thyroid carcinoma. Endokrynol Pol 2006;57(Suppl A):26-31.

19. Borgoño CA, Michael IP, Diamandis EP. Human tissue kallikreins: physiologic roles and applications in cancer. Mol Cancer Res 2004;2:257-280.

20. Paliouras M, Borgono C, Diamandis EP. Human tissue kallikreins: the cancer biomarker family. Cancer Lett 2007;249:61-79.

21. Talieri M, Mathioudaki K, Prezas $P$, et al. Clinical significance of kallikrein-related peptidase $7\left(\mathrm{KLK}_{7}\right)$ in colorectal cancer.
Thromb Haemost 2009;101:741-747.

22. Arden KC. Multiple roles of FOXO transcription factors in mammalian cells point to multiple roles in cancer. Exp Gerontol 2006;41:709-717.

23. Reagan-Shaw S, Ahmad N. The role of Forkhead-box Class O (FoxO) transcription factors in cancer: a target for the management of cancer. Toxicol Appl Pharmacol 2007;224:360368.

24. Karger S, Weidinger C, Krause K, et al. FOXO3a: a novel player in thyroid carcinogenesis? Endocr Relat Cancer 2009;16:189-199.

25. Akagi T, Luong QT, Gui D, et al. Induction of sodium iodide symporter gene and molecular characterisation of $\mathrm{HNF}_{3}$ beta/FoxA2, TTF-1 and C/EBP beta in thyroid carcinoma cells. Br J Cancer 2008;99:781-788. 